\title{
INGRESOS ECONÓMICOS EN MÉDICOS PERUANOS SEGÚN ESPECIALIDAD: UN ANÁLISIS TRANSVERSAL DE LA ENSUSALUD 2015
}

\author{
Alvaro Taype-Rondan'1 , J. Smith Torres-Roman², Percy Herrera-Añazco ${ }^{3,4}$, Carlos Alva Diaz ${ }^{5,6}$, \\ Ana Brañez-Condorena ${ }^{7}$, Miguel G. Moscoso-Porras ${ }^{7}$
}

\begin{abstract}
RESUMEN
Objetivos. Evaluar la asociación entre tener una especialidad médica y el ingreso económico mensual en médicos peruanos, y comparar los ingresos económicos entre áreas con mayor y menor densidad de médicos en Perú. Materiales y métodos. Se analizaron los datos de la Encuesta Nacional de Satisfacción de Usuarios de Salud realizada en Perú el año 2015. Esta encuesta con nivel de inferencia nacional fue realizada a médicos que laboran en establecimientos de salud de Perú. Se evaluó el ingreso económico considerando todas las actividades remuneradas del médico. Se calcularon las razones de prevalencia crudas y ajustadas (RP y RPa) y sus intervalos de confianza al 95\% (IC95\%) mediante regresiones de Poisson con varianza robusta, tomando en cuenta el muestreo complejo de la encuesta. Resultados. De 2219 médicos encuestados, se analizaron 2154 (97,0\%) observaciones. La frecuencia de ganar >S/ 5000 (1572,3 USD) mensuales fue de 29,1\% en médicos generales; $65,6 \%$ en especialistas; $63,0 \%$ en especialidades clínicas; $70,5 \%$ en especialidades quirúrgicas, y $55,7 \%$ en otras especialidades. En comparación a los médicos generales, los médicos con especialidades clínicas, quirúrgicas, y otras especialidades, tuvieron más probabilidades de ganar $>S / 5000$ mensuales $(R P a=1,44,1,49$, y 1,26 , respectivamente). La probabilidad de ganar >S/ 5000 fue mayor en quienes laboraban en departamentos con baja densidad de médicos. Conclusiones. Los ingresos económicos fueron mayores en médicos especialistas que en no especialistas. Los ingresos económicos fueron mayores en departamentos con menor densidad de médicos, lo cual puede animar a que los médicos laboren en dichos departamentos.
\end{abstract}

Palabras clave: Salarios y beneficios; Recursos humanos; Emigración e inmigración (fuente: DeCS BIREME).

\section{ECONOMIC INCOME IN PERUVIAN PHYSICIANS ACCORDING TO THE SPECIALTY: A CROSS-SECTIONAL ANALYSIS OF THE ENSUSALUD 2015}

\author{
ABSTRACT \\ Key words: Wages and benefits; Human resources; Emigration and immigration (source: MeSH NLM).

\footnotetext{
CRONICAS Centro de Excelencia en Enfermedades Crónicas, Universidad Peruana Cayetano Heredia. Lima, Perú

Facultad de Medicina, Universidad Nacional San Luis Gonzaga. Ica, Perú.

Escuela de Medicina, Universidad Peruana de Ciencias Aplicadas. Lima, Perú.

Hospital Nacional Dos de Mayo. Lima, Perú.

Unidad de Posgrado, Facultad de Medicina, Universidad Nacional Mayor de San Marcos. Lima, Perú.

Red de Eficacia Clínica y Sanitaria (REDECS), Lima, Perú.

Asociación para el Desarrollo de la Investigación Estudiantil en Ciencias de la Salud - ADIECS. Lima, Perú.

Recibido: 23/11/2016 Aprobado: 05/04/2017 En línea: 28/06/2017
}

Objectives. To evaluate the relationship between having a medical specialty and the monthly income of Peruvian doctors, and to compare the economic incomes among areas with higher and lower density of medical doctors in Peru. Materials and methods. We analyzed data of the National Satisfaction Survey of Health Users (in Spanish: ENSUSALUD) carried out in Peru in the year 2015. This survey, with a national level of inference, was performed on physicians working at health facilities in Peru. Monthly income was measured considering all paid activities of the physician. Crude and adjusted prevalence ratios (PR and aPR) and their 95\% confidence intervals $(95 \% \mathrm{Cl})$ were calculated through Poisson regression models with robust variance, taking into account the complex sampling of the survey. Results. Out of 2219 Physicians surveyed, $2154(97.0 \%)$ observations were analyzed. The probability of earning > S/5 000 (1 572.3 USD) per month was $29.1 \%$ for general practitioners; $65.6 \%$ for specialists; $63.0 \%$ for clinical specialists; $70.5 \%$ for surgeons, and $55.7 \%$ for other specialties. Compared to general practitioners, physicians with clinical, surgical, and other specialties were more likely to earn $>S / 5000$ per month $(a P R=1.44,1.49$, and 1.26 , respectively). The probability of earning $>\mathrm{S} / 5000$ was higher in those working in departments with low medical density. Conclusions. Monthly incomes were higher for specialist physicians than for non-specialists. Economic incomes were higher in departments with lower density of physicians, which may encourage physicians to work in these departments.

Citar como: Taype-Rondan A, Torres-Roman JS, Herrera-Añazco P, Alva Diaz C, Brañez-Condorena A, Moscoso-Porras AG. Ingresos económicos en médicos peruanos según especialidad: un análisis transversal dela ENSUSALUD 2015. Rev Peru Med Exp Salud Publica. 2017;34(2):183-91. doi: 10.17843/rpmesp.2017.342.2517 


\section{INTRODUCCIÓN}

El ingreso económico del médico es un factor importante que se debe considerar en las políticas de salud pública, pues los bajos ingresos están asociados a desenlaces negativos, como una menor satisfacción laboral, realizar mayores jornadas laborales ${ }^{(1)}$, realizar doble práctica laboral ${ }^{(2,3)}$, incrementar la intensidad de los servicios prestados, invertir menor tiempo por paciente ${ }^{(4)}$, renunciar al trabajo, y migrar ${ }^{(5)}$.

El ingreso económico suele ser diferente entre especialistas y no especialistas ${ }^{(6,7)}$. Dentro del grupo de especialistas, suele ser mayor entre médicos con especialidades quirúrgicas ${ }^{(6,8)}$, probablemente se deba a que estos realizan más procedimientos. En contraste, los médicos con especialidades relacionadas a la de atención primaria, como medicina familiar, suelen tener menores ingresos ${ }^{(9)}$.

En Perú se ha reportado que los médicos perciben sus ingresos como bajos, lo cual los obligaría a laborar en más de un lugar ${ }^{(10)}$. Además, los bajos ingresos parecen favorecer la intención de los médicos de migrar fuera del país ${ }^{(11,12)}$ y de no laborar fuera de la ciudad de Lima ${ }^{(13)}$, lo cual puede estar contribuyendo a la distribución inequitativa de estos profesionales en los distintos departamentos del país ${ }^{(14)}$. Sin embargo, pocos son los estudios que han estimado el sueldo de médicos peruanos, estos se han realizado en la ciudad de Lima, y no han distinguido médicos generales de especialistas ${ }^{(15,16)}$.

En este contexto, resulta importante evaluar los ingresos económicos en médicos especialistas y no especialistas, y en zonas con diferente densidad de médicos. Esto permitiría diseñar estrategias salariales para animar a los médicos a realizar ciertas especialidades necesarias para el país, o a laborar en zonas donde haya mayor demanda de médicos.

Por lo expuesto, el presente estudio tuvo dos objetivos: 1) Evaluar la asociación entre tener una especialidad médica y el ingreso económico mensual en médicos peruanos, y 2) Comparar los ingresos económicos entre áreas con mayor y menor densidad de médicos en Perú.

\section{MATERIALES Y MÉTODOS}

\section{TIPO DE ESTUDIO}

Análisis secundario de datos de la Encuesta Nacional de Satisfacción de Usuarios de Salud del año 2015 (ENSUSALUD 2015) realizada por la Superintendencia Nacional de Salud y el Instituto Nacional de Estadística e Informática (INEI). La ENSUSALUD 2015 fue un estudio transversal con inferencia nacional, que constó de seis cuestionarios usados para recopilar información de

\section{MENSAJES CLAVE}

Motivación para realizar el estudio. El ingreso económico de los médicos podría influir en la atención brindada y en la distribución irregular de estos profesionales. Además, es un tema muy poco estudiado en Perú.

Principales hallazgos. Principales hallazgos. Se analizaron datos de 2154 médicos peruanos. Solo el $29,1 \%$ de los médicos generales y el $65,6 \%$ de los especialistas ganaban más de S/ 5000 (\$1572) mensuales. Los ingresos fueron mayores entre los médicos que laboraban en departamentos con baja densidad de médicos.

Implicancias. Los ingresos de los médicos generales y especialistas son menores a los reportados en otros países. Se requieren políticas salariales para mejorar esta situación.

usuarios de servicios de salud, personal de farmacias y personal de salud ${ }^{(17)}$. Para este estudio se utilizó la base de datos del segundo cuestionario, realizado al personal médico y de enfermería, con el objetivo de conocer sus características, incluyendo su actividad laboral.

\section{POBLACIÓN Y MUESTRA}

ENSUSALUD 2015 tuvo como población al personal de salud (médicos y enfermeros) que laboran en instituciones prestadoras de servicios de salud (IPRESS) administrados por el Ministerio de Salud (MINSA), el Seguro Social (EsSalud), los hospitales de las fuerzas armadas y policiales, y las clínicas privadas.

Se realizó un muestreo de dos etapas. En la primera etapa se definió a cada departamento como un estrato. La unidad primaria de muestreo dentro de cada estrato fueron los IPRESS. En la segunda etapa se realizó un muestreo sistemático del personal de salud de cada IPRESS, de acuerdo al número de atenciones por día y la carga del evaluador técnico. El cálculo del tamaño muestral determinado para tener niveles de inferencia a nivel nacional y regional fue de 5067 profesionales de salud: 2219 médicos y 2848 enfermeros. Para el presente estudio se utilizó solo la información del personal médico que reportó información sobre sus ingresos económicos.

\section{VARIABLES}

\section{Ingreso económico mensual}

El ingreso mensual de los médicos fue recolectado mediante la pregunta: "Considerando todas sus actividades remuneradas, ¿cuál es su nivel de ingreso mensual?". Las alternativas posibles fueron: "Menor a S/ 1000"; "de S/ 1000 a S/ 2000"; "de S/ 2001 a S/ 3000"; "de S/ 3001 a S/ 4000"; "de S/ 4001 a S/ 5000", y "Más de S/ 5000". 
Para el análisis subsecuente, esta variable se dicotomizó en dos categorías: $\leq \mathrm{S} / 5000$ " y > S/ 5000. El símbolo "S/" se refiere a soles, moneda oficial del Perú. Para expresar estas cantidades en dólares, se usó el tipo de cambio promedio para el año 2015 (1 USD = S/ 3,18).

\section{Especialidad médica}

La especialidad médica fue determinada mediante dos preguntas: "¿Tiene alguna especialidad?" (Síl No) y “¿Cuál es su especialidad?" (con respuesta abierta). Los participantes fueron categorizados como "medicina general", si reportaban no tener especialidad médica; "especialidad clínica", si su especialidad estaba relacionada a actividades preventivas, diagnósticas, o terapéuticas ni quirúrgicas; "especialidad quirúrgica", si su especialidad estaba relacionada a procedimientos quirúrgicos; y "otras especialidades", si su especialidad no estaba contemplada en las otras dos categorías. Las especialidades categorizadas en cada grupo se muestran en la tabla suplementaria.

\section{Otras variables}

Otras variables utilizadas para los análisis fueron: sexo, edad (en terciles), años de labor en el sector salud (en terciles), tipo de contrato (nombrado/no nombrado), institución de trabajo (MINSA/EsSalud/Fuerzas Armadas y policía/clínicas privadas), horas laborales por semana sumando todas las actividades remuneradas (en terciles), número de labores realizadas (una/dos/tres o más), y densidad de médicos en el departamento donde labora (menor/intermedia/mayor).

El número de labores realizadas fue una variable construida a través de las preguntas: "¿Realiza labor asistencial en otra institución?" (Sí/No); “¿Realiza labor no asistencial en otro establecimiento de salud?" (Sí/No), y "¿Realiza labor docente remunerada?" (Sí/No). Se consideró "solo una actividad laboral" cuando el encuestado respondió "No" a las tres preguntas, "dos actividades laborales" cuando el encuestado respondió "Si" a solo una de las preguntas, y "tres o más actividades laborales" cuando respondió "Si" a más de una de las preguntas.

La densidad de médicos fue construida usando información de la tasa de profesionales médicos por cada diez mil habitantes para el año $2013^{(18)}$. Se categorizó a los departamentos donde laboraba el encuestado de acuerdo a su densidad en terciles: "densidad mayor" para los que tuvieron >14 médicos (Lima, el Callao, y Arequipa); "densidad intermedia" para los que tuvieron de 7 a 14 médicos (Apurímac, Ica, La Libertad, Lambayeque, Moquegua, Madre de Dios, Pasco, Tacna y Tumbes); y "densidad menor" a los que tuvieron $<7$ médicos por cada diez mil habitantes (Huancavelica,
Cusco, Huánuco, Loreto, Cajamarca, San Martín, Junín, Puno, Piura, Ucayali, Ayacucho, Ancash, y Amazonas).

\section{ANÁLISIS ESTADÍSTICO}

Todos los análisis fueron realizados con el software estadístico Stata 14.0 (StataCorp LP, College Station, TX, USA). Las características generales de los participantes fueron presentadas utilizando frecuencias absolutas y relativas, así como medias \pm desviaciones estándar.

Se evaluaron los factores asociados a tener un ingreso mensual >S/ 5000 usando modelos lineales generalizados. Se obtuvieron las probabilidades predichas y las razones de prevalencia crudas y ajustadas (RP y $\mathrm{RPa}$ ) con sus respectivos intervalos de confianza al 95\% (IC95\%) usando modelos con familia de distribución de Poisson y función de enlace logarítmica. Además, se realizaron análisis estratificados por especialidad médica para evaluar la asociación entre el nivel de densidad de médicos y el ingreso económico >S/ 5000.

Para todos los análisis se consideró el tipo de muestreo realizado por ENSUSALUD. La base de datos fue configurada para un muestreo complejo, donde las unidades primarias de muestreo fueron los conglomerados dentro de cada estrato. Además, para el análisis estratificado por especialidad, las estimaciones de los errores estándar fueron calculadas considerando a cada especialidad como una subpoblación y usando un método centrado, pues en uno de los casos solo existía un conglomerado por estrato.

Adicionalmente, se graficó la probabilidad de tener un ingreso mensual >S/ 5000 por cada una de las especialidades médicas que tuvieran 20 o más observaciones.

\section{ASPECTOS ÉTICOS}

Los datos de este estudio fueron obtenidos de una base de datos pública (http://iinei.inei.gob.pe/microdatos/). Los encuestadores de ENSUSALUD realizaron un consentimiento verbal a cada uno de los participantes, indicando que la encuesta era anónima y voluntaria. Solo se recolectó información de los participantes voluntarios.

\section{RESULTADOS}

\section{DESCRIPCIÓN DE LA POBLACIÓN}

De los 2219 médicos encuestados, se excluyó la información de 65 que no reportaron sus ingresos 
económicos, quedando $2154(97,0 \%)$ para el análisis final. Los médicos excluidos no presentaron diferencias en cuanto a su edad, especialidad, ni institución laboral frente a los no incluidos, pero sí presentaron con mayor frecuencia las siguientes características: tener $\geq 23$ años de tiempo laboral y laborar en departamentos del segundo quintil de densidad de médicos.
De los 2154 sujetos evaluados, 553 (25,7\%) fueron mujeres; la edad promedio fue $46,3 \pm 11,4$ años; el número de años laborados en el sector salud promedio fue de 17,0 $\pm 10,2$ años; las horas laborales promedio por semana fueron 51,1 $\pm 15,2$ horas, y $1429(66,3 \%)$ reportaron tener alguna especialidad. Todas las variables analizadas fueron estadísticamente diferentes entre médicos generales y especialistas (Tabla 1).

Tabla 1. Características de médicos generales y especialistas encuestados, ENSUSALUD 2015.

\begin{tabular}{|c|c|c|c|c|}
\hline Característica n(\%) & $\begin{array}{c}\text { Total } \\
\mathrm{N}=2154\end{array}$ & $\begin{array}{c}\text { Generales } \\
\mathrm{N}=725\end{array}$ & $\begin{array}{c}\text { Especialistas } \\
\mathrm{N}=1429\end{array}$ & $\begin{array}{c}\text { Valor } \\
\mathbf{p}\end{array}$ \\
\hline Especialidad & & & & $<0,001$ \\
\hline Médico general & $725(33,7)$ & $725(100,0)$ & $0(0,0)$ & \\
\hline Especialidad clínica & $752(34,9)$ & $0(0,0)$ & $752(52,6)$ & \\
\hline Especialidad quirúrgica & $580(26,9)$ & $0(0,0)$ & $580(40,6)$ & \\
\hline Otras especialidades & $97(4,5)$ & $0(0,0)$ & $97(6,8)$ & \\
\hline Sexo & & & & $<0,001$ \\
\hline Varón & $1601(74,3)$ & $486(67,0)$ & $1115(78,0)$ & \\
\hline Mujer & $553(25,7)$ & $239(33,0)$ & $314(22,0)$ & \\
\hline Edad & & & & $<0,001$ \\
\hline 24 a 40 años & $768(35,7)$ & $444(61,2)$ & $324(22,7)$ & \\
\hline 41 a 53 años & $712(33,1)$ & $154(21,2)$ & $558(39,1)$ & \\
\hline 54 a 88 años & $674(31,3)$ & $127(17,5)$ & $547(38,3)$ & \\
\hline Años laborando en el sector salud & & & & $<0,001$ \\
\hline 1 a 11 años & $748(34,7)$ & $463(63,9)$ & $285(19,9)$ & \\
\hline 12 a 22 años & $721(33,5)$ & $154(21,2)$ & $567(39,7)$ & \\
\hline 23 a 55 años & $685(31,8)$ & $108(14,9)$ & $577(40,4)$ & \\
\hline Tipo de contrato & & & & $<0,001$ \\
\hline No nombrado & $1304(60,5)$ & $513(70,8)$ & $791(55,3)$ & \\
\hline Nombrado & $850(39,5)$ & $212(29,2)$ & $638(44,7)$ & \\
\hline Institución & & & & 0,001 \\
\hline MINSA & $992(46,1)$ & $365(50,3)$ & $627(43,9)$ & \\
\hline EsSalud & $1000(46,4)$ & $298(41,1)$ & $702(49,1)$ & \\
\hline Fuerzas Armadas y Policía & $29(1,4)$ & $15(2,1)$ & $14(1,0)$ & \\
\hline Clínicas & $133(6,1)$ & $47(6,5)$ & $86(6,0)$ & \\
\hline Horas de trabajo semanales & & & & $<0,001$ \\
\hline$<41$ horas semanales & $742(34,5)$ & $340(46,9)$ & $402(28,1)$ & \\
\hline 41 a 56 horas semanales & $746(34,6)$ & $216(29,8)$ & $530(37,1)$ & \\
\hline 57 a 130 horas semanales & $666(30,9)$ & $169(23,3)$ & $497(34,8)$ & \\
\hline Número de labores que realiza & & & & $<0,001$ \\
\hline Solo realiza una labor & $881(40,9)$ & $471(65,0)$ & $410(28,7)$ & \\
\hline Realiza dos labores & $837(38,9)$ & $191(26,3)$ & $646(45,2)$ & \\
\hline Realiza tres o más labores & $436(20,2)$ & $63(8,7)$ & $373(26,1)$ & \\
\hline Densidad de médicos por departamento & & & & $<0,001$ \\
\hline Mayor & $709(32,9)$ & $173(23,9)$ & $536(37,5)$ & \\
\hline Intermedia & $688(31,9)$ & $220(30,3)$ & $468(32,8)$ & \\
\hline Menor & $757(35,2)$ & $332(45,8)$ & $425(29,7)$ & \\
\hline
\end{tabular}




\section{INGRESO ECONÓMICO}

El porcentaje de médicos que reportó ganar >S/ 5000 mensuales (1572,3 USD) fue $29,1 \%$ en médicos generales; $65,6 \%$ en médicos con alguna especialidad; $63,0 \%$ en especialidades clínicas; $70,5 \%$ en especialidades quirúrgicas, y $55,7 \%$ en otras especialidades. En el modelo ajustado, los médicos con especialidades clínicas, quirúrgicas, y otras especialidades, tuvieron más probabilidades de ganar
>S/ 5000 mensuales en comparación a los médicos generales $(\mathrm{RPa}=1,44,1,49$, y 1,26 , respectivamente). Al evaluar los factores asociados a ganar $>S / 5000$ mensuales, se encontró que este se asocia con tener alguna especialidad, ser de sexo masculino, tener más años laborando en el sector salud, laborar en EsSalud o en clínicas (en comparación con quienes laboran en MINSA), laborar más horas por semana, realizar múltiples labores, y laborar en departamentos con menor densidad de médicos (Tabla 2).

Tabla 2. Factores asociados a tener un ingreso económico mayor a S/ 5000 (1572,3 USD) mensuales considerando todas sus actividades remuneradas

\begin{tabular}{|c|c|c|c|c|}
\hline \multirow{2}{*}{ Factores } & \multicolumn{2}{|c|}{$\begin{array}{c}\text { Ingreso económico mensual } \\
>S / 5000\end{array}$} & \multirow{2}{*}{$\begin{array}{c}\text { Modelo crudo } \\
\text { RP (IC95\%) }\end{array}$} & \multirow{2}{*}{$\begin{array}{c}\text { Modelo ajustado * } \\
\text { RPa (IC95\%) }\end{array}$} \\
\hline & $\begin{array}{c}\text { No } \\
\text { N (\%) }\end{array}$ & $\begin{array}{c}\text { Sí } \\
\mathbf{N}(\%) \\
\end{array}$ & & \\
\hline \multicolumn{5}{|l|}{ Especialidad } \\
\hline Médico general & $514(70,9)$ & $211(29,1)$ & Ref & Ref \\
\hline Especialidad clínica & $278(37,0)$ & $474(63,0)$ & $2,17(1,82-2,58)$ & $1,44(1,23-1,67)$ \\
\hline Especialidad quirúrgica & $171(29,5)$ & $409(70,5)$ & $2,42(2,01-2,92)$ & $1,49(1,28-1,74)$ \\
\hline Otras especialidades & $43(44,3)$ & $54(55,7)$ & $1,91(1,53-2,39)$ & $1,26(1,03-1,55)$ \\
\hline \multicolumn{5}{|l|}{ Sexo } \\
\hline Varón & $631(39,4)$ & $970(60,6)$ & Ref & Ref \\
\hline Mujer & $375(67,8)$ & $178(32,2)$ & $0,53(0,46-0,61)$ & $0,74(0,65-0,85)$ \\
\hline \multicolumn{5}{|l|}{ Edad } \\
\hline 24 a 40 años & $508(66,1)$ & $260(33,9)$ & Ref & Ref \\
\hline 41 a 53 años & $269(37,8)$ & $443(62,2)$ & $1,84(1,59-2,12)$ & $1,04(0,92-1,17)$ \\
\hline 54 a 88 años & $229(34,0)$ & $445(66,0)$ & $1,95(1,67-2,27)$ & $1,09(0,93-1,28)$ \\
\hline \multicolumn{5}{|l|}{ Años laborando en el sector salud } \\
\hline 1 a 11 años & $515(68,9)$ & $233(31,1)$ & Ref & Ref \\
\hline 12 a 22 años & $272(37,7)$ & $449(62,3)$ & $2,00(1,72-2,33)$ & $1,35(1,17-1,56)$ \\
\hline 23 a 55 años & $219(32,0)$ & $466(68,0)$ & $2,18(1,86-2,56)$ & $1,39(1,16-1,65)$ \\
\hline \multicolumn{5}{|l|}{ Tipo de contrato } \\
\hline No nombrado & $662(50,8)$ & $642(49,2)$ & Ref & Ref \\
\hline Nombrado & $344(40,5)$ & $506(59,5)$ & $1,21(1,07-1,37)$ & $1,09(0,98-1,21)$ \\
\hline \multicolumn{5}{|l|}{ Institución } \\
\hline MINSA & $543(54,7)$ & $449(45,3)$ & Ref & Ref \\
\hline EsSalud & $387(38,7)$ & $613(61,3)$ & $1,35(1,12-1,64)$ & $1,33(1,16-1,51)$ \\
\hline Fuerzas Armadas y Policía & $18(62,1)$ & $11(37,9)$ & $0,84(0,46-1,54)$ & $0,95(0,66-1,36)$ \\
\hline Clínicas & $58(43,6)$ & $75(56,4)$ & $1,25(0,94-1,65)$ & $1,30(1,05-1,61)$ \\
\hline \multicolumn{5}{|l|}{ Horas de trabajo semanales } \\
\hline 2 a 40 horas semanales & $527(71,0)$ & $215(29,0)$ & Ref & Ref \\
\hline 41 a 56 horas semanales & $299(40,1)$ & $447(59,9)$ & $2,07(1,69-2,54)$ & $1,31(1,13-1,52)$ \\
\hline 57 a 130 horas semanales & $180(27,0)$ & $486(73,0)$ & $2,52(2,09-3,04)$ & $1,42(1,22-1,64)$ \\
\hline \multicolumn{5}{|l|}{ Número de labores que realiza } \\
\hline Solo realiza una labor & $661(75,0)$ & $220(25,0)$ & Ref & Ref \\
\hline Realiza dos labores & $274(32,7)$ & $563(67,3)$ & $2,69(2,18-3,33)$ & $1,83(1,51-2,23)$ \\
\hline Realiza otras dos o más labores & $71(16,3)$ & $365(83,7)$ & $3,35(2,71-4,15)$ & $2,04(1,67-2,49)$ \\
\hline \multicolumn{5}{|l|}{ Densidad de médicos por departamento } \\
\hline Mayor & $402(56,7)$ & $307(43,3)$ & Ref & Ref \\
\hline Intermedia & $274(39,8)$ & $414(60,2)$ & $1,39(1,10-1,75)$ & $1,27(1,11-1,46)$ \\
\hline Menor & $330(43,6)$ & $427(56,4)$ & $1,30(1,06-1,60)$ & $1,34(1,17-1,52)$ \\
\hline
\end{tabular}

* Este modelo fue ajustado por todas las variables que figuran en la tabla 
Al comparar las especialidades que tuvieron, por lo menos, 20 encuestados, los médicos especialistas en anestesiología y otorrinolaringología tuvieron mayor probabilidad de tener ingresos mensuales $>S / 5000$, mientras que los especialistas en diagnóstico por imágenes y oncología presentaron las probabilidades más bajas (Figura 1).

\section{INGRESO ECONÓMICO SEGÚN DENSIDAD DE MÉDICOS DEL DEPARTAMENTO}

Al evaluar la asociación entre la densidad de médicos en el departamento donde labora el encuestado y percibir ingresos $>S / 5000$, se encontró que en los departamentos con "menor densidad" los médicos generales, especialistas clínicos y especialistas quirúrgicos tuvieron ingresos económicos significativamente mayores $(\mathrm{RPa}=$ $1,68,1,30$, y 1,29 respectivamente) a los que laboraban en departamentos con "mayor densidad" (Tabla 3 ).

\section{DISCUSIÓN}

La probabilidad de percibir un ingreso económico >S/5000 (1572,3 USD) fue mayor en los médicos especialistas en comparación con los médicos generales, y fue similar entre los que tuvieron especialidades clínicas y quirúrgicas. Además, esta probabilidad fue mayor en los médicos varones, aquellos que laboran en EsSalud o clínicas, aquellos que han laborado durante más años, aquellos que laboran más horas semanales, aquellos que realizan más labores, y aquellos que laboran en departamentos con menor densidad de médicos.

En el presente análisis, se reporta que para el 2015 el 62,3\% de médicos generales ganaron >S/ 4000 (1257,9 USD), pero $29,1 \%$ ganaron >S/ 5000 (1572,3 USD) mensuales, por lo cual la mediana de ingresos se ubicaría entre S/ 4000 y S/ 5000. Entre los médicos especialistas, $65,6 \%$ reporta ganar >S/ 5000 mensuales, por lo cual, la mediana sería mayor a dicho monto. Estas cifras son mayores a las estimaciones realizadas para Perú por dos estudios previos para el 2004 en Lima: S/ 1919 (548,3 USD) ${ }^{(15)}$ y S/ 1930 (567,6 USD) ${ }^{(16)}$, y son mucho mayores a la remuneración mínima vital para Perú, que es de S/ 850 (267,3 USD).

Sin embargo, los ingresos encontrados son menores a los ingresos mensuales reportados en países de la Organisation for Economic Co-operation and Development en el periodo 2011-2015: de 1425,6 a 21 752,8 USD para médicos generales, y de 1974,9 a 21 259,7 USD para especialistas (19). Asimismo, serían menores a las expectativas salariales de S/ 8000 .

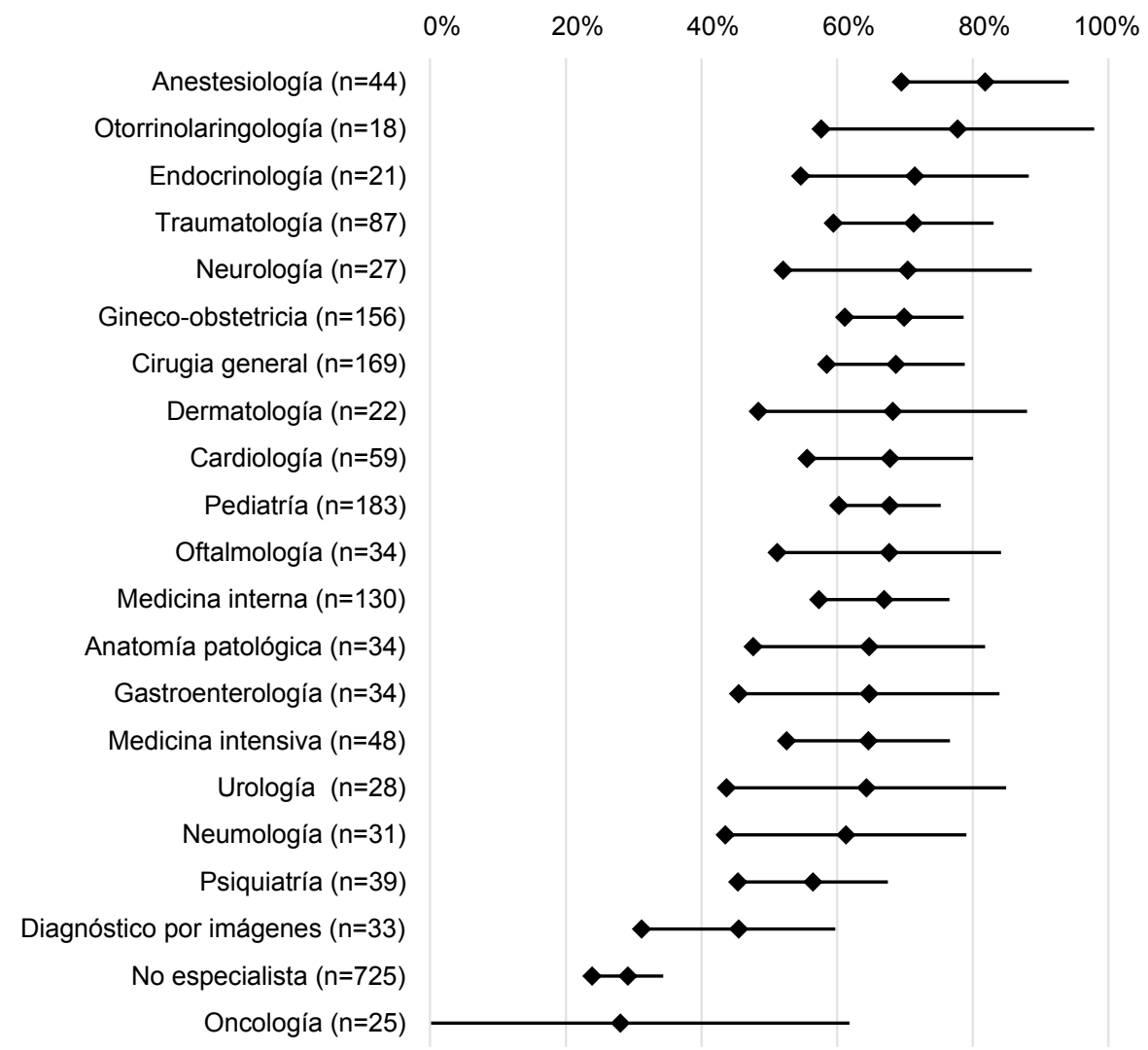

Figura 1. Porcentajes de médicos que tienen un ingreso económico >S/ 5000 (1572,3 USD) mensuales sumando todas sus actividades remuneradas: frecuencia e IC95\% (Intervalos de confianza al 95\%) 
Tabla 3. Asociación entre densidad de médicos por departamento y tener un ingreso económico >S/ 5000 (1572,3 USD) mensuales, en cuatro estratos según especialidad

\begin{tabular}{|c|c|c|c|c|c|c|c|c|}
\hline \multirow{2}{*}{$\begin{array}{l}\text { Densidad de } \\
\text { médicos por } \\
\text { departamento* }\end{array}$} & \multicolumn{2}{|c|}{ Médicos generales } & \multicolumn{2}{|c|}{ Especialidad clínica } & \multicolumn{2}{|c|}{ Especialidad quirúrgica } & \multicolumn{2}{|c|}{ Otras especialidades } \\
\hline & $\%(n / N)$ & $\mathrm{RPa}(\mathrm{IC} 95 \%)^{\dagger}$ & $\%(n / N)$ & $\operatorname{RPa}(\mathrm{IC} 95 \%)^{\dagger}$ & $\%(n / N)$ & $\mathrm{RPa}(\mathrm{IC} 95 \%)^{\dagger}$ & $\%(n / N)$ & $\mathrm{RPa}(\mathrm{IC} 95 \%)^{\dagger}$ \\
\hline Mayor & $15,6(27 / 173)$ & Ref & $50,8(163 / 321)$ & Ref & $55,9(104 / 186)$ & Ref & $44,8(13 / 29)$ & Ref \\
\hline Intermedia & $29,1(64 / 220)$ & $1,72(1,02-2,91)$ & $73,9(173 / 234)$ & $1,28(1,09-1,50)$ & $79,6(152 / 191)$ & $1,30(1,11-1,53)$ & $58,1(25 / 43)$ & $0,95(0,64-1,40)$ \\
\hline Menor & $36,1(120 / 332)$ & $1,68(1,07-2,64)$ & $70,1(138 / 197)$ & $1,30(1,08-1,55)$ & $75,4(153 / 203)$ & $1,29(1,08-1,55)$ & $64,0(16 / 25)$ & $1,01(0,68-1,51)$ \\
\hline
\end{tabular}

*División según terciles de densidad.

${ }^{\dagger}$ Ajustado por sexo, edad, tiempo laborando en el sector salud, ser nombrado, institución, horas laboradas por semana, y número de labores que realiza

(2580,6 USD) que reportaron internos de medicina de una universidad de Lima en el año $2007^{(20)}$.

Esta diferencia entre los ingresos de médicos peruanos y extranjeros, así como entre las expectativas salariales y el ingreso final de los médicos peruanos, resulta importante, pues puede influir en la satisfacción del médico y su decisión de migrar fuera del país (10-12). Por ello, resulta necesario que se desarrollen políticas sanitarias para disminuir estas brechas. Un paso importante fue el Decreto Legislativo 1153 aprobado el 2013, que establecía una nueva política remunerativa basada en mérito, desempeño y riesgo según el nivel de atención (21).

Los ingresos económicos fueron mayores en los médicos especialistas en comparación con los médicos generales, resultados que son similares a los encontrados en otros estudios ${ }^{(8,9,22,23)}$. Esto puede deberse a que los distintos sistemas de salud dan una mayor valoración al tiempo de los especialistas, que se traduce en mayor remuneración por hora laborada; así como a una mayor oferta laboral, lo que les permite laborar en más lugares y más horas semanales.

Entre los médicos especialistas, la probabilidad de tener un ingreso económico >S/ 5000 fue mayor en médicos de especialidades quirúrgicas, similar a lo encontrado en reportes en Estados Unidos (9,24). Sin embargo, dichos estudios evaluaron el desenlace numéricamente, mientras que en la ENSUSALUD se evaluó en rangos, lo cual dificulta realizar una adecuada comparación.

Al comparar los ingresos entre especialistas, los anestesiólogos y traumatólogos estuvieron entre las especialidades mejor pagadas, lo cual coincide con los reportes del $2015^{(24)}$ y $2016^{(9)}$ de Estados Unidos, y con otros países como Suiza y Francia ${ }^{(22)}$. Otra especialidad con altos ingresos fue endocrinología que, paradójicamente, fue una de las especialidades con menores ingresos en Estados Unidos ${ }^{(9,24)}$. Entre los especialistas con menores ingresos se encuentra la especialidad de psiquiatría, lo cual coincide con reportes anteriores de Estados Unidos ${ }^{(8)}$ y Canadá ${ }^{(25)}$. Sin embargo, estos resultados deben ser tomados con cautela debido al escaso número de médicos de cada especialidad encuestados.

Otro factor asociado a tener mayores ingresos económicos fue laborar en EsSalud o en clínicas (en comparación con laborar en MINSA). Esta diferencia entre MINSA y EsSalud ha sido reportado anteriormente para varias profesiones de la salud (26), y resulta de interés, en tanto que puede desanimar a los médicos de trabajar para MINSA, que es el principal prestador de servicios de salud en el país.

Las diferencias encontradas en ingresos económicos entre especialidades pueden estar desalentando a los médicos a realizar ciertas especialidades en las que hay especial déficit, como psiquiatría, o pueden estar alentando a los médicos con dichas especialidades a migrar o laborar en establecimientos privados donde podrían tener mayores ingresos. Por ello, resulta necesario implementar políticas salariales equitativas que aseguren una remuneración idónea para las especialidades básicas y aquellas en las que existe déficit de recursos humanos.

Un factor de interés fue ser varón, similar a lo encontrado en otros estudios. Un reciente reporte reporta una brecha económica en profesionales médicos de 13 especialidades en Estados Unidos a favor de los varones ${ }^{(27)}$. Esta inequidad se suma a diferencias en el acceso a educación, salud y participación política, que se observa especialmente en países de medianos y bajos ingresos ${ }^{(28)}$. Estos resultados pueden deberse a una menor valoración del trabajo de las mujeres, o a menores oportunidades para alcanzar cargos de mayores ingresos. Esta situación debe ser estudiada a profundidad para generar marcos institucionales de solución, como asignar un porcentaje mínimo de mujeres en cargos dirigenciales, y flexibilizar las licencias parentales.

Muchos departamentos del Perú tienen una baja densidad de médicos ${ }^{(29)}$ y un déficit de especialistas ${ }^{(14)}$, 
posiblemente debido a ciertas desventajas, como menos comodidades, falta de mejoras salariales, escasas oportunidades de formación y capacitación, entre otras.

Los médicos que laboraron en departamentos con "mayor densidad" de médicos tuvieron menor ingreso económico, lo cual coincide con reportes de otros países ${ }^{(8,30)}$. Esto puede deberse a una mayor oferta de médicos, dado que los departamentos con mayor densidad incluyen a Lima, la ciudad con mayor número de escuelas de Medicina en Perú. Adicionalmente, los departamentos con mayor densidad de médicos son los que tienen un mayor costo de vida, de manera que según la Encuesta Nacional de Hogares para el 2012, la canasta básica mensual fue de S/ 320,6 en los departamentos de mayor densidad, S/ 263,8 en los de densidad intermedia, y S/ 239,7 en los de menor densidad, por lo cual, las diferencias relativas en los ingresos económicos serían aun más grandes.

En el grupo de "otras especialidades" (no clínicas ni quirúrgicas), la densidad de médicos pareció no afectar significativamente el ingreso económico. Sin embargo, la interpretación de este resultado debe ser realizada con cautela debido al escaso número de muestra en este grupo (97 médicos).

A pesar de estos resultados, un estudio realizado en 782 médicos recién colegiados de Lima encontró que solo 7,0\% planeaba trabajar fuera de Lima ${ }^{(13)}$. Por ello, resulta necesario difundir las ventajas de laborar en otros departamentos, como son los mayores ingresos económicos. Esto puede ser especialmente efectivo en los médicos que realizan el Servicio Rural Urbano Marginal en Salud en distintos departamentos del Perú, en quienes la idea de mayores ingresos puede animar a seguir laborando en dichos departamentos por algunos años más. Por ejemplo, un estudio en médicos de la ciudad de Ayacucho mostró que la intención de laborar en áreas rurales aumentaba en $21 \%$ al incrementar su salario en $50 \%$, y aumentaba en $77 \%$ al incrementar su salario en $75 \%{ }^{(31)}$.
El estudio presenta ciertas limitaciones: 1) El ingreso económico fue analizado de manera categórica y no numérica, por lo cual no se puede evaluar adecuadamente la distribución del mismo. 2) La base de datos analizada no tiene información de médicos que laboran en establecimientos del primer nivel de atención, donde labora el $42 \%$ de los médicos del MINSA ${ }^{(29)}$, quienes por trabajar en un menor nivel de complejidad podrían tener menores ingresos económicos. 3) La ENSUSALUD ha realizado su muestreo en base a la profesión (médicos y enfermeros), pero no en base a las categorías de especialidad de los médicos. Sin embargo, dado que los médicos fueron escogidos aleatoriamente, esto no debería ser un sesgo para analizar los grupos de especialidad. Asimismo, 4) Es posible que algunos resultados no significativos sean producto de un error tipo 2, por el limitado tamaño de muestra.

A pesar de estas limitaciones, este es, a nuestro conocimiento, el primer estudio que evalúa los ingresos económicos de médicos en Perú, uno de los pocos en Latinoamérica, y que aporta evidencia que puede utilizarse para estrategias que busquen mejorar la oferta y demanda de médicos en el país.

En conclusión, según los datos de la ENSUSALUD 2015, los ingresos económicos fueron mayores en médicos especialistas en comparación a los médicos generales. El ingreso fue mayor en departamentos con menor densidad de médicos, lo cual puede animar a los médicos a laborar en dichos departamentos.

Contribuciones de autoría: ATR planteó el tema de estudio, ATR y MGMP realizaron el análisis estadístico, todos los autores redactaron el manuscrito y aprobaron su versión final.

Conflictos de interés: los autores declaran no tener conflictos de interés con respecto al presente artículo

Financiamiento: el presente estudio ha sido autofinanciado

\section{REFERENCIAS BIBLIOGRÁFICAS}

1. McGuire TG, Pauly MV. Physician response to fee changes with multiple payers. J Health Econ. 1991;10(4):385-410.

2. Moghri J, Mohammad A, Rashidian A, Sari AA. Physician Dual Practice: A Descriptive Mapping Review of Literature. Iran J Public Health 2016;45(3):278-88.

3. Ferrinho P, Van Lerberghe W, Fronteira I, Hipólito F, Biscaia A. Dual practice in the health sector: review of the evidence. Hum Resour Health 2004;2(1): 14 .
4. US Government Accountability Office. Testimony before the Subcommittee on Health, Committee on Energy and Commerce, House of Representatives: Medicare physician payments: trends in service utilization, spending, and fees prompt consideration of alternative payment approaches [citado el 21 de septiembre del 2016]. Disponible en: http://www.gao.gov/new.items/ d061008t.pdf.

5. Astor A, Akhtar T, Matallana MA, Muthuswamy V, Olowu FA, Tallo V, et al. Physician migration: views from professionals in Colombia, Nigeria, India, Pakistan and the Philippines. Soc Sci Med. 2005;61(12):2492-500. doi: 10.1016/j.socscimed.2005.05.003.

6. Phillips RL, Dodoo MS, Petterson S, Xierali I, Bazemore A, Teevan B, et al. Specialty and Geographic Distribution of the Physician Workforce: What Influences Medical Student and Resident Choices? Washington (DC): Robert Graham Center; 2009 [citado el 10 de octubre del 2016]. Disponible en: http://www.graham-center.org/ $\mathrm{dam} / \mathrm{rgc} /$ documents/publications- 
reports/monographs-books/Specialtygeography-compressed.pdf.

7. Morgan $\mathrm{P}$, Everett CM, Humeniuk KM, Valentin VL. Physician assistant specialty choice: Distribution, salaries, and comparison with physicians. JAAPA. 2016;29(7):46-52. doi: 10.1097/01. JAA.0000484301.35696.16.

8. Shih YCT, Konrad TR. Factors associated with the income distribution of full time physicians: A quantile regression approach. Health Serv Res. 2007;42(5):1895-925.

9. Peckham C. Medscape Physician Compensation Report 2016 [Internet]. Medscape. 2016 [citado el 6 de julio de 2016 ]. Disponible en: http://www.medscape.com/features/ slideshow/compensation/2016/ public/overview\#page $=1$.

10. Jumpa M, Jan S, Mills A. The role of regulation in influencing income-generating activities among public sector doctors in Peru. Hum Resour Health. 2007;5(1):1.

11. Pereyra-Elías R, Cassana A, Mezones-Holguín E, Mayta-Tristán P. Diferencias según género en la intención de emigración en médicos recién egresados. An Fac med. 2013;74(3):211-6.

12. Mayta-Tristán P, Dulanto-Pizzorni A. Prevalencia y factores asociados con la intención de emigración en internos de medicina de una universidad pública, Lima 2007. Rev Peru Med Exp Salud Publica. 2008;25(3):274-8.

13. Mayta-Tristán P, Mejia CR, RiegaLopez P, Rojas-Mezarina L, Posso M, Mezones-Holguín E. Proyección de trabajo en el interior del país y factores asociados en médicos recién colegiados de Lima, Perú 2010. Rev Peru Med Exp Salud Publica. 2011;28(2):186-93.

14. Zevallos L, Pastor R, Moscoso B. Oferta y demanda de médicos especialistas en los establecimientos de salud del Ministerio de Salud: brechas a nivel nacional, por regiones y tipo de especialidad. Rev Peru Med Exp Salud Publica. 2011;28(2):177-85.

15. Webb R, Valencia S. Human resources in public health and education in Peru. A new social contract for Peru: an agenda for improving education, health care, and the social safety net Washington DC: World Bank; 2005.

16. Yamada Fukusaki G. Retornos a la educación superior en el mercado laboral: vale la pena el esfuerzo?
[Internet] Lima: Centro de Investigación de la Universidad del Pacífico; CIES, 2007. [citado el 16 de noviembre del 2016]. Disponoble en: http://old.cies.org.pe/files/DyP/ dyp31.pdf.

17. Superintendencia Nacional de Salud. Encuestas de Satisfacción a nivel Nacional (ENSUSALUD 2015). Lima: SUSALUD; 2015. Disponible en: http://portales.susalud.gob.pe/ web/portal/239.

18. Ministerio de Salud. Compendio Estadistico: Informacion de Recursos Humanos del Sector Salud Perú-2013, Lima: MINSA; 2014 [citado el 14 de noviembre del 2016]. Disponible en: http://observatorio.inforhus.gob.pe/ publicaciones/bibliograficos/libro18/ compendio201301.pdf.

19. Taype-Rondán Á, Luna-Porta L, Quispe OM. El ingreso económico como incentivo para la migración médica: explorando los números. Salud Publica Mex. 2016;58(3):337-8.

20. Mayta-Tristán P, Dulanto-Pizzorni A, Miranda JJ. Low wages and brain drain: an alert from Peru. Lancet. 2008;371(9624):1577. doi: 10.1016/ S0140-6736(08)60685-6.

21. Perú, Ministerio de Salud. Decreto Legislativo que regula la Política Integral de Compensaciones y Entregas Económicas del personal de la salud al servicio del Estado. Decreto Legislativo 1153. El Peruano. 12 de septiembre del 2013 [citado el 10 de noviembre del 2016]. Disponible en: http://www. minsa.gob.pe/dggdrh/poli_remu/ compendio/politica/DL\%201153.pdf.

22. Fujisawa R, Lafortune G. The Remuneration of General Practitioners and Specialists in 14 OECD Countries: What are the Factors Influencing Variations across Countries? OECD Health Working Papers: OECD Publishing; 2008.

23. Cheng TC, Scott A, Jeon SH, Kalb G, Humphreys J, Joyce C. What factors influence the earnings of general practitioners and medical specialists? Evidence from the medicine in Australia: balancing employment and life survey. Health Econ. 2012;21(11):1300-17. doi: 10.1002/ hec. 1791

24. Peckman C. Medscape Physician Compensation Report 2015 [Internet]. Medscape. 2015 [citado el 6 de julio de 2016]. Disponible en: http://www.medscape.com/features/ slideshow/compensation/2015/ public/overview\#page $=1$.

25. Canadian Institute for Health Information. National Physician Database, 2014-2015 Data Release [Internet]. Ontario: CIHI; 2016. [citado el 10 de noviembre de 2016]. Disponible en: https://secure.cihi.ca/estore/productFamily.htm?pf=PFC3268\&lan$\mathrm{g}=\mathrm{en} \& \mathrm{media}=0$

26. Pardo K, Andia M, Rodriguez A, Pérez W, Moscoso B. Remuneraciones, beneficios e incentivos laborales percibidos por trabajadores del sector salud en el Perú: análisis comparativo entre el Ministerio de Salud y la Seguridad Social, 2009. Rev Peru Med Exp Salud Publica. 2011;28(2):34251.

27. Desai T, Ali S, Fang X, Thompson W, Jawa P, Vachharajani T. Equal work for unequal pay: the gender reimbursement gap for healthcare providers in the United States. Postgrad Med J 2016;92(1092):571-5. doi: 10.1136/ postgradmedj-2016-134094.

28. Country Profiles: Peru [Internet]. World Economic Forum. [citado el 10 de noviembre de 2016]. Disponible en: http://reports.weforum.org/ global-gender-gap-report-2016/ economies/\#economy=PER.

29. Cabrera HEG, Sánchez JDC, Altamirano JL. Segunda Medición de las Metas Regionales de Recursos Humanos para la Salud Perú 20072015. Lima: Ministerio de Salud; 2013 [citado el 20 de julio de 2016]. Disponible en: http://observatorio. inforhus.gob.pe/publicaciones/ bibliograficos/libro15/I-15.pdf.

30. Reschovsky JD, Staiti AB. Physician incomes in rural and urban America. Issue Brief Cent Stud Health Syst Change. 2005;(92):1-4.

31. Miranda JJ, Diez-Canseco F, Lema C, Lescano AG, Lagarde M, Blaauw D, et al. Stated preferences of doctors for choosing a job in rural areas of Peru: a discrete choice experiment. PloS One. 2012;7(12):e50567. doi: 10.1371/ journal.pone.0050567.

\footnotetext{
Correspondencia: Álvaro Taype-Rondan

Dirección: CRONICAS Centro de Excelencia en Enfermedades Crónicas, Universidad Peruana Cayetano Heredia, Lima, Perú. Avenida Armendáriz 497, Miraflores, Lima 18, Perú Teléfono: (+51) 2416978

Correo electrónico: alvaro.taype.r@gmail.com
} 